polyarthritis, ${ }^{5}$ dactylitis, ${ }^{1}$ enthesitis, ${ }^{1}$ psoriasis, ${ }^{1}$ uveitis ${ }^{1}$ and inflammatory bowel disease. ${ }^{1}$ All patients were therapeutic-naive for glucocorticostreroids, DMARDs or TNF- $\alpha$ inhibitors.

Methods: First, we performed examination of the sacroiliac joint with X-ray, to exclude those patients, who already had radiographic lesions. Then an MRI was performed in the following sequences: T2- weighted STIR for the bone marrow oedema (BME) and T1-weighted sequence for the fat metaplasia (FM). The HDP SPECT/CT was used within one week to examine the sacroiliac joint. Thereafter, the MRI images were fused with HDP SPECT/CT images. On the MRI images the $\mathrm{BME}$ (active lesion) and FM (chronic lesion), on the CT scans the sclerotic lesions (SCL, chronic lesion) were drawn manually as volume of interest (VOI). Uninvolved cortical areas were drawn on the different modality slices as reference region (ref). Then, we determined the isotope (99mTc-labelled HDP) uptake of the different lesions and areas.

Results: Four active sacroileitis and five chronic sacroileitis without active lesions were diagnosed according to the MRI results. On the other 8 patient's sacroiliac joints images (MRI, scintigraphy, CT scans), no inflammation-related lesions were observed. The MRI and HDP SPECT-CT findings were $100 \%$ concordant. The isotope uptake of $\mathrm{BME}$ was the highest, the radioisotope uptake of sclerotic lesions was moderate, whereas the isotope uptake of FM lesions was not different from the HDP uptake of reference regions.

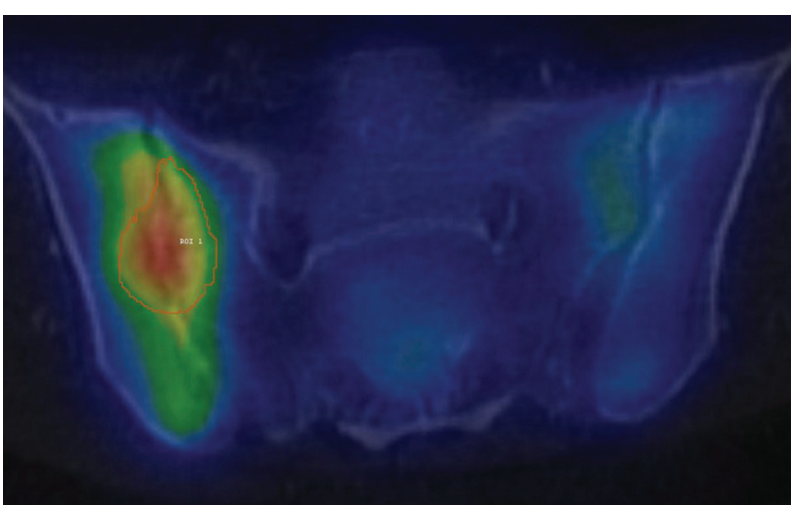

Conclusions: According to the initial results, the different MRI lesions have different isotope uptake, which suggests, that the HDP SPECT/CT can distinguish the early and chronic stage of axial SpA from chronic lesions. Thank to whole body imaging technique we can have further information about disease activity and extent. The presented data are the first of our prospective study, and examinations of new patients are still in progress and we plan to investigate our SpA patients in remission to explore the utility of this new method in subclinical activity assessment. We also plan to investigate the corner lesions of the spine to find other potential uses of the HDP SPECT-CT imaging in SpA.

Disclosure of Interest: None declared

DOI: 10.1136/annrheumdis-2018-eular.2222

\section{SAT0670 DYNAMIC THIOL/DISULFIDE HOMEOSTASIS AS A NOVEL OXIDATIVE MARKER IN BEHÇET'S DISEASE}

S. Can Sandikci ${ }^{1}$, S. Colak ${ }^{2}$, A. Omma ${ }^{2}$, M.E. Enecik ${ }^{3}$, Z. Ozbalkan²,

S. Neselioglu ${ }^{4}$, O. Erel ${ }^{4} .{ }^{1}$ Rheumatology, Ankara Diskapi Yildirim Beyazit Training And Research Hospital; ${ }^{2}$ Rheumatology, Ankara Numune Training and Research Hospital, ankara; ${ }^{3}$ rheumatology, Mersin City Hospital, Mersin; ${ }^{4}$ Biochemistry, Yildirim Beyazit University, Ankara, Turkey

Background: Behçet's disease $(\mathrm{BD})$ is a relapsing systemic inflammatory disorder of unknown etiology. ${ }^{1}$

Objectives: In this study, we aimed to evaluate the relationship between the thioldisulfide balance and disease activity and organ involvement in BD.

Methods: One hundred fifty (150) patients with BD and 100 age-gender matched healty controls were included in the study. Disease activity was assessed with the BD Current Activity Form (BDCAF) score. Serum levels of native thiol (NT), total thiol (TT), and disulfide were measured and the disulfide/native thiol, disulfide/total thiol and native thiol/total thiol levels were calculated for the patient and control groups.

Results: NT, TT, NT/TT values of the BD patients were significantly lower than those of the control group. The disulfide/NT, disulfide/TT values of BD patients were higher compared to the control group and the disulfide value of the BD group was slightly higher compared to the control group (table 1). No correlation was determined between thiol levels and disease activity and organ involvement in BD.
Abstract SAT0670 - Table 1. Laboratory results of the BD and the control group

\begin{tabular}{lccc}
\hline & BD & HC & p \\
\hline Native Thiol $(\mathrm{SH})(\mu \mathrm{mol} / \mathrm{l})$ & 357.93 & $453.01 \pm 60.8$ & $\mathbf{0 . 0 0 0 ^ { * }}$ \\
& \pm 76.65 & & \\
Total Thiol $(\mu \mathrm{mol} / \mathrm{l})$ & 398.57 & 490.29 & $\mathbf{0 . 0 0 0 ^ { * }}$ \\
& \pm 78.33 & \pm 58.48 & \\
Disulfide $(\mu \mathrm{mol} / \mathrm{l})(\mathrm{SS})$ & $20.32 \pm 8.19$ & $18.64 \pm 7.87$ & $\mathbf{0 . 1 0 9}$ \\
Disulfide/native thiol $\mathbf{x 1 0 0}$ & $5.93 \pm 2.76$ & $4.25 \pm 1.99$ & $\mathbf{0 . 0 0 0 ^ { * }}$ \\
Disulfide/total thiol x100 & $5.2 \pm 2.13$ & $3.86 \pm 1.69$ & $\mathbf{0 . 0 0 0 ^ { * }}$ \\
Native thiol/total thiol & $89.6 \pm 4.26$ & $92.28 \pm 3.38$ & $\mathbf{0 . 0 0 0 ^ { * }}$ \\
$\mathbf{x} 100$ & & & \\
\hline
\end{tabular}

${ }^{*} p<0,05$

Conclusions: In patients with Behcet's disease, the dynamic thiol-disulfide homeostasis balance shifted towards disulfide formation due to thiol oxidation. I may be used as a novel marker in BD because it is easy, practical, fully automated and relatively inexpensive.

Acknowledgements: The authors thank staff of Ankara Numune Training and Research Hospital, Department of Rheumatology for their generous friendly assistance in every step of this study.

Disclosure of Interest: None declared

DOI: 10.1136/annrheumdis-2018-eular.5278

\section{SAT0671 INITIAL DEVELOPMENT OF A WHOLE-BODY MAGNETIC RESONANCE IMAGING INFLAMMATION INDEX FOR ACTIVE DISEASE OF PERIPHERAL JOINTS AND ENTHESES IN PATIENTS WITH INFLAMMATORY ARTHRITIS}

S. Krabbe ${ }^{1,2}$, P. Bird ${ }^{3}$, I. Eshed ${ }^{4}$, V. Foltz ${ }^{5}$, F. Gandjbakhch ${ }^{5,6}$, D. Glinatsi ${ }^{1}$, J. L. Jaremko ${ }^{7}$, R.G. Lambert ${ }^{7}$, W.P. Maksymowych ${ }^{8}$, A.J. Mathew ${ }^{9}$, S.J. Pedersen ${ }^{1}$, R. Poggenborg ${ }^{1}$, M.S. Stoenoiu ${ }^{10}$, P.G. Conaghan ${ }^{11}$, C.E. Althoff ${ }^{12}$, C. Peterfy ${ }^{13}$, K.-G. Hermann ${ }^{14}$, M. Østergaard ${ }^{1,2}$ on behalf of OMERACT MRI Working Group. ${ }^{1}$ Copenhagen Center for Arthritis Research, Center for Rheumatology and Spine Diseases, Rigshospitalet, ${ }^{2}$ Department of Clinical Medicine, Faculty of Health and Medical Sciences, University of Copenhagen, Copenhagen, Denmark; ${ }^{3}$ Division of Medicine, University of New South Wales, Sydney, Australia; ${ }^{4}$ Department of Diagnostic Imaging, Sheba Medical Center, Affiliated to the Sackler School of Medicine, Tel Aviv University, Tel Aviv, Israel; ${ }^{5}$ Hôpitaux Universitaires Pitié Salpêtrière; ${ }^{6}$ Paris 6 University, GRC-UPMC 08, Pierre Louis Institute of Epidemiology and Public Health, Paris, France; ${ }^{7}$ Department of Radiology and Diagnostic Imaging, University of Alberta; ${ }^{8} \mathrm{CaRE}$ (Canadian Research Education) Arthritis and Department of Medicine, University of Alberta, Edmonton, Canada; ${ }^{9}$ Department of Clinical Immunology and Rheumatology, Christian Medical College, Vellore, India; ${ }^{10}$ Cliniques Universitaires Saint-Luc, Institut de Recherche Expérimentale et Clinique (IREC), Université catholique de Louvain, Brussels, Belgium; ${ }^{11}$ Faculty of Medicine and Health, University of Leeds, UK; ${ }^{12}$ Institut und Klinik für Radiologie CCM und CVK Charité - Universitätsmedizin, Berlin, Germany, ${ }^{13}$ Spire Sciences, Inc., San Francisco, USA; ${ }^{14}$ Department of Radiology, Arthritis Imaging Research Group, University Hospital Charité, Berlin, Germany

Background: Magnetic resonance imaging (MRI) allows objective assessment of inflammation in peripheral joints and entheses. MRI scoring systems have until now focused on assessing specific parts of the musculoskeletal system in detail, e.g. the Rheumatoid Arthritis MRI Scoring System (RAMRIS), which is applied to wrist and metacarpophalangeal joints and adjacent tendon sheaths. The interest in a whole-body MRI approach is growing as modern MRI scanners now permit whole-body scanning within an acceptable time frame, and future improvements in MRI hardware and pulse sequences are expected to improve scan time and image resolution further.

Objectives: To develop a whole-body MRI scoring system for inflammation of peripheral joints and entheses and to investigate its feasibility and reliability.

Methods: Definitions of the key pathologies and locations for assessment have been agreed upon in the OMERACT MRI Working Group ${ }^{1}$. In a first round in June 2017, 9 readers (AJM/DG/FG/IE/MØ/PB/SJP/SK/WPM) scored MR images of 2 patients with spondyloarthritis using a draft web-based scoring system. Results were discussed and the scoring system was slightly modified. Hereafter, in a second round in October 2017, $14 \mathrm{MRI}$ readers (3 musculoskeletal radiologist (IE/ $\mathrm{JLJ} / \mathrm{RGL}$ ) and 11 rheumatologists with varying exposure to MRI (AJM/DG/FG/ $\mathrm{MS} / \mathrm{M} \varnothing / \mathrm{PB} / \mathrm{RP} / \mathrm{SJP} / \mathrm{SK} / \mathrm{VF} / \mathrm{WPM})$, scored 5 similar patients by the modified scoring system. Using a semiquantitative scale 0-3 (none/mild/moderate/severe), synovitis and osteitis were scored separately for 83 joints, and soft tissue inflammation and osteitis were scored separately for 33 entheses. Discrepancies between readers were discussed during an online meeting to obtain consensus, to train inexperienced readers, and to identify potential pitfalls when applying the scoring system. 